\title{
The topology of critical sets of some ordinary differential operators
}

\author{
Nicolau C. Saldanha and Carlos Tomei
}

June 30,2018

Dedicated to Djairo Figueiredo, with affection and admiration.

\begin{abstract}
We survey recent work of Burghelea, Malta and both authors on the topology of critical sets of nonlinear ordinary differential operators. For a generic nonlinearity $f$, the critical set of the first order nonlinear operator $F_{1}(u)(t)=u^{\prime}(t)+f(u(t))$ acting on the Sobolev space $H_{p}^{1}$ of periodic functions is either empty or ambient diffeomorphic to a hyperplane. For the second order operator $F_{2}(u)(t)=-u^{\prime \prime}(t)+f(u(t))$ on $H_{D}^{2}$ (Dirichlet boundary conditions), the critical set is ambient diffeomorphic to a union of isolated parallel hyperplanes. For second order operators on $H_{p}^{2}$, the critical set is not a Hilbert manifold but is still contractible and admits a normal form. The third order case is topologically far more complicated.
\end{abstract}

Keywords: Sturm-Liouville, nonlinear differential operators, infinite dimensional manifolds.

MSC-class: Primary 34L30, 58B05, Secondary 34B15, 46 T05.

\section{Introduction}

We survey recent work of Burghelea, Malta and both authors on the topology of critical sets $C$ of (nonlinear, ordinary) differential operators $F$. Our approach is geometric, in the sense that we study the geometry and topology of $C$ and its image $F(C)$ with the purpose of understanding the function $F$. Pioneering examples of this point of view for nonlinear differential equations are the results on the Laplacian coupled to a special nonlinearity by Ambrosetti and Prodi ([1), interpreted as properties of a global fold by Berger and Podolak (2]). 
We begin this paper with a two dimensional example in which most of our claims can be followed visually. We then consider differential operators of increasing difficulty. For generic nonlinearities $f: \mathbb{R} \rightarrow \mathbb{R}$, set $F_{1}(u)=u^{\prime}+f(u)$ on the Sobolev space $H_{p}^{1}$ of periodic functions $([12])$, with critical set $C_{1}$. We show that if $C_{1}$ is not empty then there is a global diffeomorphism in the domain of $F_{1}$ converting $C_{1}$ into a hyperplane. There are two parts in the argument. First, we prove that the homotopy groups of $C_{1}$ are trivial. We then use the fact that, under very general conditions, (weak) homotopically equivalent infinite dimensional Hilbert manifolds are actually diffeomorphic. For some classes of nonlinearities, we achieve a global normal form for $F_{1}$.

We proceed to the second order operator $F_{2, D}(u)=-u^{\prime \prime}+f(u)$ acting on $H_{D}^{2}([0, \pi])$, the Sobolev space of functions satisfying Dirichlet boundary conditions (4]). The critical set $C_{2, D}$ now splits into connected components $C_{2, D, m}$, one for each positive integer value of $m$ for which $-m^{2}$ is in the interior of the image of $f^{\prime}$. Again, there exists a diffeomorphism taking each $C_{2, D, m}$ to a hyperplane. The proof of the triviality of homotopy groups of $C_{2, D, m}$ requires a very different strategy than the first order case.

Second order operators on $H_{p}^{2}$ (the space of periodic functions) are more complicated, in the sense that the critical set is not a manifold, but with suitable hypothesis is still contractible and admits a normal form ([5]). In brief, the critical set looks like a union of a hyperplane and infinitely many cones, with nonregular points at the vertices. Nonregular points in the critical set correspond to potentials $h(t)=f^{\prime}(u(t)), u \in H_{p}^{2}$, for which the kernel of the linear operator $v \mapsto-v^{\prime \prime}+h(t) v$ is a subspace of dimension 2 of $H_{p}^{2}$. The diffeomorphism of $H_{p}^{2}$ through which the critical set achieves its normal form takes the set of nonregular critical points to a disjoint union of linear subspaces of codimension 3 .

The third order case is yet more complicated: consider the set $C_{3, p}^{*}$ of pairs $\left(h_{0}, h_{1}\right)$ for which the kernel of the linear operator $u \mapsto u^{\prime \prime \prime}+h_{1} u^{\prime}+h_{0} u$ is a subspace of $H_{p}^{3}$ of dimension 3. In the final section we show that the space $C_{3}^{*}$ is homotopically equivalent to $X_{I}$, the set of closed locally convex curves in the sphere $\mathbb{S}^{2}$ with a prescribed initial base point and direction. It turns out that the space $X_{I}$ has three connected components ([9]), two of them having a rich algebraic topological structure, not equivalent to any finitely generated CW-complex ([16], [18, [19], [20]).

The authors acknowledge the support of CNPq, Faperj and Finep. 


\section{A finite dimensional example}

Consider

$$
\begin{aligned}
F: \mathbb{C} & \rightarrow \mathbb{C} \\
z & \mapsto z^{7}+5 \bar{z}^{4}+z
\end{aligned}
$$

which is clearly a smooth (but not analytic) function from the plane $\mathbb{R}^{2}$ to itself. In this section, we identify $\mathbb{C}$ and $\mathbb{R}^{2}$ indiscriminately. How many solutions has the equation $F(z)=0$ ?

Our approach to the question $([11,17])$ breaks into a few steps. First, we compute the critical set $C$ of $F$ by searching for points in the plane in which the Jacobian $D F$ is not invertible. Some numerical analysis reveals that $C$ consists of two simple curves $C_{i}$ and $C_{o}$ bounding disks $(0,0) \in D_{i} \subset D_{o}$. A finer inspection verifies that, as expected from Whitney's classical theorems on planar singularity theory $(21])$, the generic critical point is a fold, i.e., after local changes of variables the function takes the form $(x, y) \mapsto\left(x, y^{2}\right)$ close to the origin. Also, nonfold points are cusps, with local normal form $(x, y) \mapsto\left(x, y^{3}+x y\right)$. Again, with the help of some computation, one finds out that the curves $C_{i}$ and $C_{o}$ have five and eleven cusps, respectively. What may be more informative is the geometry of the images $F\left(C_{i}\right)$ and $F\left(C_{o}\right)$, presented in the picture below.
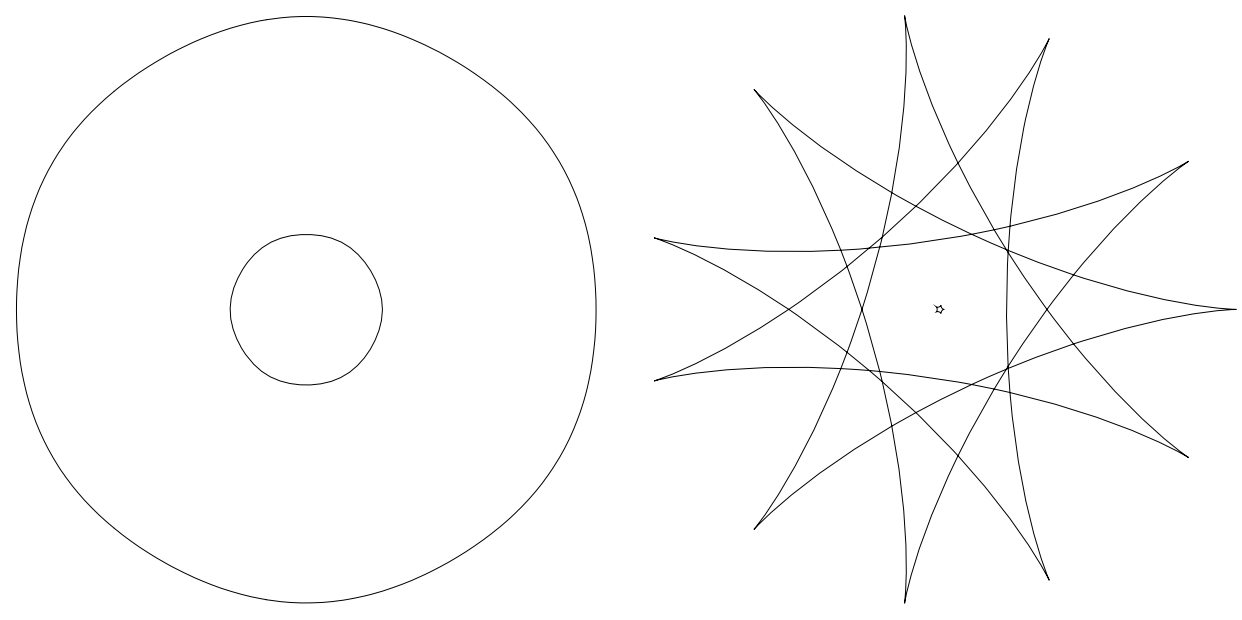

Figure 1: The critical set of the function $F(z)=z^{7}+5 \bar{z}^{4}+z$ and its image.

We now count preimages. Simple estimates suffice to prove that $F$ is proper, and that, for sufficiently large $\left(w_{1}, w_{2}\right) \in \mathbb{R}^{2}$, the equation $F(x, y)=\left(w_{1}, w_{2}\right)$ has seven solutions. From properness, points in the same connected component of the complement of $F(C)$ have the same number of pre-images. Now, consider two points $p$ and $q$ in components sharing a boundary arc of (images of) folds. From 
the normal form of a function at a fold point, the number of preimages of $p$ and $q$ under $F$ differ by two. The sense of folding indicates that the number of preimages increases by two whenever one gets closer to the origin when trespassing an arc of images of folds. Adding up, from the knowledge that points in the unbounded connected component of the complement of $F(C)$ have seven preimages, we learn the number of preimages of each component. Thus, the origin has 17 preimages. In a nutshell, the number of preimages of a point under a function $F$ may become large when the image of the critical set $C$ intersects itself abundantly.

In the following sections, we shall consider functions between separable infinite dimensional Hilbert spaces. In many cases, the critical set turns out to be surprisingly simple.

\section{The first order operator}

We consider the differential equation

$$
u^{\prime}(t)+f(u(t))=g(t), \quad u(0)=u(\pi)
$$

where the unknown $u$ is a real function and $f: \mathbb{R} \rightarrow \mathbb{R}$ is a smooth nonlinearity. In the spirit of the example in the previous section, we define the operator

$$
\begin{aligned}
F_{1}: H_{p}^{1} & \rightarrow L^{2} \\
u & \mapsto u^{\prime}+f(u)
\end{aligned}
$$

where $H_{p}^{1}=H_{p}^{1}([0,1] ; \mathbb{R})=H^{1}\left(\mathbb{S}^{1} ; \mathbb{R}\right)$ is the Sobolev space of periodic functions with weak derivative in $L^{2}=L^{2}([0,1] ; \mathbb{R})$. It is easy to verify that the differential

$$
D F_{1}(u) v=v^{\prime}+f^{\prime}(u) v
$$

is a Fredholm operator of index 0 and therefore $u$ belongs to the critical set $C_{1} \subset H_{p}^{1}$ of $F_{1}$ if and only if the equation

$$
v^{\prime}(t)+f^{\prime}(u(t)) v(t)=0, \quad v(0)=v(1)
$$

admits a nontrivial solution $v$, i.e.,

$$
C_{1}=\left\{u \in H_{p}^{1} \mid \phi_{1}(u)=0\right\}, \quad \phi_{1}(u)=\int_{0}^{1} f^{\prime}(u(t)) d t .
$$

An equivalent spectral interpretation for $C_{1}$ is

$$
C_{1}=\left\{u \in H_{p}^{1} \mid 0 \in \sigma\left(D F_{1}(u)\right)\right\} .
$$

It is not hard to see $\left([12)\right.$ that $D F_{1}(u)$ has a unique real eigenvalue. We assume that $f^{\prime \prime}$ has isolated roots which are distinct from the roots of $f^{\prime}$ : this implies 
that 0 is a regular value of $\phi_{1}$ and therefore that $C_{1}$ is a smooth hypersurface in $H_{p}^{1}$. An $\mathbb{H}$-manifold is a manifold modeled on the separable infinite dimensional Hilbert space $\mathbb{H}: C_{1}$ is an $\mathbb{H}$-manifold. With these hypothesis, the topology of $C_{1}$ is trivial ([12]):

Theorem 1 Assume $C_{1}$ to be nonempty. Then $C_{1}$ is path connected and contractible. Furthermore, there is a diffeomorphism from $H_{p}^{1}$ to itself taking $C_{1}$ to a hyperplane.

A natural finite dimensional counterpart of this theorem is false. Indeed, let

$$
C_{1}^{n}=\left\{u \in \mathbb{R}^{n} \mid \phi_{1}^{n}(u)=0\right\}, \quad \phi_{1}^{n}(u)=\sum_{k} f^{\prime}\left(u_{k}\right) .
$$

For $f(x)=x^{3}-x, C_{1}^{n}$ is a sphere. Thus, our theorem goes hand in hand with the well known facts that in an infinite dimensional Hilbert space $\mathbb{H}$, the unit sphere is contractible and there is a diffeomorphism of $\mathbb{H}$ to itself taking the unit sphere to a hyperplane.

The rest of this section outlines the proof of theorem 1. First, one proves ([13]) that the homotopy groups $\pi_{k}\left(C_{1}\right)$ are trivial. We consider only $k=0$, i.e., path connectedness of $C_{1}$, the other cases being similar. This is done first in the space $C^{0}$ (with the sup norm): more precisely, set

$$
C_{1}^{0}=\left\{u \in C^{0}([0,1]) \mid \phi_{1}(u)=0\right\} ;
$$

it is easier to build a homotopy in the sup norm, since it is weaker. Take two functions $u_{0}(t)$ and $u_{1}(t)$ in $C_{1}^{0}$ so that

$$
\int_{0}^{1} f\left(u_{0}(t)\right) d t=\int_{0}^{1} f\left(u_{1}(t)\right) d t=0 .
$$

Let $s \in[0,1]$ be the parameter for the desired homotopy $\mathbf{h}(s, t)$ : we want $\mathbf{h}(0, t)=$ $u_{0}(t), \mathbf{h}(1, t)=u_{1}(t), \mathbf{h}(s, \cdot) \in C_{1}^{0}$. Define a discontinuous function $\mathbf{h}_{0}:[0,1] \times$ $[0,1] \rightarrow \mathbb{R}$ by $\mathbf{h}_{0}(s, t)=u_{0}(t)$ if $(s, t) \in A \subset[0,1] \times[0,1]$ and $\mathbf{h}_{0}(s, t)=u_{1}(t)$ otherwise, where $A$ looks like the set in figure 2 , for each $s, A_{s}=\{t \mid(s, t) \in A\}$ is a rather uniformly distributed subset of $[0,1]$ of measure $1-s$. For each $s$, $\phi_{1}\left(\mathbf{h}_{0}(s, \cdot)\right) \approx 0$. The function $H$ equals $\mathbf{h}_{0}$ except on a set of very small measure, where it is defined so as to obtain a continuous $\mathbf{h}$ for which $\phi_{1}(\mathbf{h}(s, \cdot))=0$.

In order to prove that the homotopy groups $\pi_{k}\left(C_{1}\right)$ are also trivial, we use the following theorem ([4]) with $Y=H_{p}^{1}, X=C^{0}([0,1]), M=C_{1}^{0}, N=C_{1}$ and $i$ the inclusion.

Theorem 2 Let $X$ and $Y$ be separable Banach spaces. Supose $i: Y \rightarrow X$ is a bounded, injective map with dense image and $M \subset X$ a smooth, closed submanifold of finite codimension. Then $N=i^{-1}(M)$ is a smooth closed submanifold 


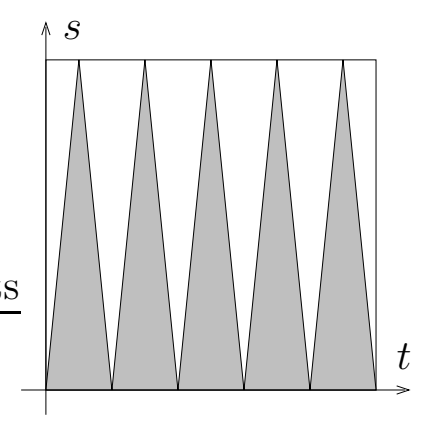

Figure 2: The set $A$ (shaded).

of $Y$, and the restrictions $i: Y-N \rightarrow X-M$ and $i:(Y, N) \rightarrow(X, M)$ are homotopy equivalences.

Since $C_{1}$, being an $\mathbb{H}$-manifold, is homotopy equivalent to a $\mathrm{CW}$-complex, weak contractibility implies contractibility and $C_{1}$ is contractible (and, by theorem 2, $C_{1}^{0}$ is contractible too). The connected components of the complement of $C_{1}$ are also contractible. Indeed, let $h: \mathbb{S}^{k} \rightarrow H_{p}^{1}-C_{1}$ be a continuous map; since $H_{p}^{1}$ is obviously contractible, $h$ can be extended to $\mathbf{h}: \mathbb{B}^{k+1} \rightarrow H_{p}^{1}$. Since $C_{1}$ is contractible, $\mathbf{h}$ can be redefined to take each connected component of $\mathbf{h}^{-1}\left(H_{p}^{1}-C_{1}\right)$ not touching $\mathbb{S}^{k}$ to $C_{1}$. A normal vector to $C_{1}$ can then be used to push the image of $\mathbf{h}$ away from $C_{1}$, yielding $\mathbf{h}: \mathbb{B}^{k+1} \rightarrow H_{p}^{1}-C_{1}$. Thus, the homotopy groups $\pi_{k}\left(H_{p}^{1}-C_{1}\right), k \geq 1$, are trivial and therefore the connected components of $H_{p}^{1}-C_{1}$ are contractible. To complete the proof of theorem 1, we use the following result (4]):

Theorem 3 Suppose $f:\left(V_{1}, \partial V_{1}\right) \rightarrow\left(V_{2}, \partial V_{2}\right)$ is a smooth homotopy equivalence of $\mathbb{H}$-manifolds with boundary, $K_{2} \subset V_{2} \backslash \partial V_{2}$ a closed submanifold of finite codimension and $K_{1}=f^{-1}\left(K_{2}\right)$. Suppose also that $f$ is transversal to $K_{2}$ and the maps $f: K_{1} \rightarrow K_{2}$ and $f: V_{1}-K_{1} \rightarrow V_{2}-K_{2}$ are homotopy equivalences. Then there exists a diffeomorphism between $\left(V_{1} ; \partial V_{1}, K_{1}\right)$ and $\left(V_{2} ; \partial V_{2}, K_{2}\right)$ which is homotopic to $f$ as maps of triples.

Set $V_{1}=H_{p}^{1}$ and consider the functional $\phi_{1}: H_{p}^{1} \rightarrow \mathbb{R}$ defined above. Write $V_{2}=L^{2}([0,1])=\langle 1\rangle \oplus\langle 1\rangle^{\perp}$, where $\langle 1\rangle \cong \mathbb{R}$ is the vector space of constant functions and $\langle 1\rangle^{\perp}$, the space of functions $v$ of average $\bar{v}$ equal to zero, is a hyperplane in $V_{2}$. Set $f: V_{1} \rightarrow V_{2}$ to be $f(u)=\left(\phi_{1}(u), 0\right)$. Set $K_{2}=\langle 1\rangle^{\perp}$ so that the critical set $C_{1}$ equals $K_{1}=f^{-1}\left(K_{2}\right)$, and the genericity condition on $f$ ensures that $C_{1}=K_{1}$ is a hypersurface of $V_{1}$ and $f$ is transversal to $K_{2}$. Notice that $\partial V_{1}=\partial V_{2}=\emptyset$. Since $K_{1}=C_{1}$ is contractible, we obtain that $f$ is a homotopy equivalence between $K_{1}$ and $K_{2}$. Similarly, since the connected components of 
$V_{1}-K_{1}$ are contractible, $f: V_{1}-K_{1} \rightarrow V_{2}-K_{2}$ is a homotopy equivalence and Theorem 1 follows.

The proofs of theorems 2 and 3 are rather technical and shall not be discussed here. A simple spinoff is the following density result which may be of independent interest.

Theorem 4 Let $X$ be a Banach space, $V \subset X$ a dense (linear) subspace and $M \subset X$ a finite codimension submanifold. Then the intersection $V \cap M$ is dense in $M$.

\section{More on $C_{1}$ and $F_{1}\left(C_{1}\right)$}

For the first order differential operator $F_{1}$, generically, it is easy to prove that the critical $C_{1}$ stratifies by complexity of the singularities, which are infinite dimensional counterparts of the familiar Morin singularities (14], [12]). In a nutshell, the Morin singularity is the generic situation for a germ from $\left(\mathbb{R}^{n}, 0\right)$ to itself whose Jacobian at 0 has a one dimensional kernel. There is essentially one elementary Morin singularity in each dimension, with very simple normal forms: the fold $x \mapsto x^{2}$, the cusp $(x, y) \mapsto\left(x, y^{3}+x y\right)$, the swallowtail $(x, y, z) \mapsto$ $\left(x, y, z^{4}+y z^{2}+x z\right)$, the butterfly $(x, y, z, t) \mapsto\left(x, y, z, t^{5}+z t^{3}+y t^{2}+x t\right), \ldots$ One should take into account Morin singularities arising by taking cartesian products: the fold $x \mapsto x^{2}$ embeds into the (non-elementary) fold $(x, y) \mapsto\left(x^{2}, y\right)$. Even in infinite dimensions, Morin singularites are always cartesian products of an elementary singularity (for which one defines a natural order, given by the degree of the top polynomial of its normal form) and the identity mapping with the appropriate codimension ([12]; also [7] for infinite dimensional cusps).

What is the topology of the finer strata? For the first order differential operator $F_{1}$ with a generic nonlinearity $f$, the subset $\Sigma_{2}$ of critical points which are nonfold points again has trivial topology. The proof of this statement begins with the following result:

Theorem 5 For a generic smooth nonlinearity $f: \mathbb{R} \rightarrow \mathbb{R}$, there is a diffeomorphism from $H_{p}^{1}$ to itself taking the sets $C_{1}$ and $\Sigma_{2}$ of $F_{1}$ respectively to the zero level of the functional $\phi_{1}$ and to the zero level of the pair $\left(\phi_{1}, \phi_{1,2}\right)$, where

$$
\phi_{1,2}(u)=\int_{0}^{1} f^{\prime \prime}(u(t)) d t .
$$

Consider now a vector valued extension of the argument used to prove that the homotopy groups of $C_{1}$ are trivial ([13]). Let $M$ be a compact, finite dimensional manifold with a smooth Riemannian metric inducing a normalized measure $\mu$, so that $\mu(M)=1$. Let $V$ a separable Banach space of continuous real valued 
functions on $M$, which is closed under multiplication by functions of $C^{\infty}(M)$ (here, multiplication in $C^{\infty}(M) \times V \rightarrow V$ is continuous). For a smooth function $f_{n}: M \times \mathbb{R} \rightarrow \mathbb{R}^{n}$ define $N_{n}: V \rightarrow \mathbb{R}^{n}$ as the average of the Nemytskiu operator associated to $f$,

$$
N_{n}(v)=\int_{M} f_{n}(m, v(m)) d \mu .
$$

Let $\Pi_{k}: \mathbb{R}^{n} \rightarrow \mathbb{R}^{k}$ be the projection on the first $k$ coordinates. We say that 0 is a strong regular value of $N_{n}$ if 0 is a regular value of each composition $N_{k}=\Pi_{k} \circ F_{n}$. Finally, let $Z_{k}=N_{k}^{-1}(0)$ : if 0 is a strong regular value of $N_{n}$, the sets $Z_{k}$ are nested submanifolds of $V$ of codimension $k$.

Theorem 6 Suppose 0 is a strong regular value of $N_{n}$ as above. Then the levels $Z_{k}$ are contractible. Moreover, there is a global homeomorphism $\Psi: V \rightarrow V$ taking each $Z_{k}$ to a closed linear subspace of $V$ of codimension $k$. If $V$ is a Hilbert space, $\psi$ can be taken to be a diffeomorphism.

Thus, for a generic nonlinearity, the zero levels of $\phi_{1}$ and $\left(\phi_{1}, \phi_{1,2}\right)$ are ambient diffeomorphic to nested subspaces of codimension 1 and 2, and we obtain the topological triviality of $C_{1}$ (again) and of $\Sigma_{2}$. Theorem 5 is necessary because the set of nonfold points is not described naturally in terms of zero levels of vector Nemytskil operators. This is the reason for which we do not know how to address topological properties of higher order strata.

The image $F\left(C_{1}\right)$ clearly depends on the nonlinearity. We list a few facts ([12]), some of which had been known ([10]).

1. Suppose $f$ is strictly increasing, onto the interval $(a, b)$. Then $F_{1}$ is a diffeomorphism onto the strip

$$
\left\{w \in L^{2} \mid a<\langle w, 1\rangle<b\right\} .
$$

2. If $f$ is strictly convex, $F_{1}$ is a global fold. If $f$ is generic, and all critical points of $F_{1}$ are folds, then $F_{1}$ is a global fold. There are nonconvex linearities for which $F_{1}$ is a global fold.

3. If $f$ is a generic polynomial of even degree, positive leading coefficient, taking negative values, then $F_{1}$ has cusps.

4. Suppose $f: \mathbb{R} \rightarrow \mathbb{R}$ is proper, with derivative $f^{\prime}$ assuming both signs and third derivative with isolated roots assuming only one sign. Then $F_{1}$ is a global cusp, in the sense that diffeomorphisms on $H_{p}^{1}$ and $L^{2}$ convert $F_{1}$ to the normal form

$$
\begin{aligned}
\tilde{F}_{1}: \mathbb{R}^{2} \times \mathbb{H} & \rightarrow \mathbb{R}^{2} \times \mathbb{H} \\
(x, y ; v) & \mapsto\left(x, y^{3}+x y ; v\right)
\end{aligned}
$$


The last example establishes a conjecture by Cafagna and Donati ([6]) on the global topology of operators associated to the nonlinearity $f(x)=a x+b x^{2}+$ $c x^{2 k+1}, a \geq 0, a^{2}+b^{2}>0$ and $c<0$.

From the examples, the number of preimages of an operator associated to a generic nonlinearity given by a polynomial of degree less than or equal to three is bounded by the degree of the polynomial. This is false for polynomials of degree four. The counterexample in [12] was obtained by adjusting coefficients of the nonlinearity in order to obtain a butterfly in the critical set of $F_{1}$. Singularity theory combined with a degree theoretic argument imply the existence of a point with six preimages, five of which are near the butterfly. Both nonlinearity and special point were computed numerically.

On a related note, Pugh conjectured that the equation

$$
u^{\prime}(t)=a_{k}(t)(u(t))^{k}+\cdots+a_{1}(t) u(t)+a_{0}(t), \quad u(1)=u(0)
$$

would have at most $k$ solutions. The conjecture was essentially verified by Smale for $k=2$ and $k=3$ if $a_{3}(t)>0$ for all $t$. The general conjecture was proved false by Lins Neto ([8]). Our example has the special feature that $a_{i}$ is constant in $t$ for $i>0$.

\section{The second order case, Dirichlet conditions}

Let $H_{D}^{2}=H_{D}^{2}([0, \pi], \mathbb{R})$ be the Sobolev space of functions with second weak derivative in $L^{2}=L^{2}([0, \pi] ; \mathbb{R})$, satisfying Dirichlet boundary conditions and for a smooth nonlinearity $f$ now consider the operator

$$
\begin{aligned}
F_{2, D}: H_{D}^{2} & \rightarrow L^{2} \\
u & \mapsto-u^{\prime \prime}+f(u)
\end{aligned}
$$

with differential given by $D F_{2, D}(u) v=-v^{\prime \prime}+f^{\prime}(u) v$. Again, by Fredholm theory, the critical set $C_{2, D}$ of $F_{2, D}$ consists of functions $u \in H_{D}^{2}$ for which $D F_{2, D}$ has zero in the spectrum.

For a more explicit description of $C_{2, D}$, define the fundamental solution $v_{1}$,

$$
-v_{1}^{\prime \prime}+f^{\prime}(u) v_{1}=0, \quad v_{1}(0)=0, v_{1}^{\prime}(0)=1,
$$

and consider the (continuously defined) argument $\omega(t)$ of the vector $\left(v_{1}^{\prime}(t), v(t)\right)$ for which $\omega(0)=0$. Notice the implicit dependence of $\omega$ on $v_{1}$, which in turn depends on $u$. Then

$$
C_{2, D}=\left\{u \in H_{D}^{2} \mid \omega(\pi) / \pi \in \mathbb{Z}\right\} .
$$


Thus, there must be different connected components of $C_{2, D}$ for each value of $\omega(\pi)=m \pi$. Said differently, the fact that the eigenvalue 0 of $D F_{2, D}(u)$ may be the $m$-th eigenvalue in the spectrum (counting from below) induces different critical components of $F_{2, D}$. Define the functional $\phi_{2, D}(u)$ as the value of the argument at $\pi$ of the fundamental solution $v_{1}$ associated to $u$ and consider subsets $C_{2, D, m}$ which partition $C_{2, D}$, given by the $m \pi$-levels of $\phi_{2, D}$. For a generic set of nonlinearities $f$, the set $C_{2, D, m}$ is nonempty if and only if the number $-m^{2}$ belongs to the interior of the image of $f^{\prime}$ : in this case, $C_{2, D, m}$ is path connected and contractible.

We want to prove that arbitrary functions from a sphere $\mathbb{S}^{k}$ to $C_{2, D, m}$ are homotopic to constants. Theorem [6 is not the appropriate tool anymore, among other reasons because the functional $\phi_{2, D}$ depends on the derivative $u^{\prime}$. From the topological theorems of section 3 , it suffices to control the deformation in the sup norm. Contractibility then implies a global change of variables in $H_{D}^{2}$ which flattens the connected components $C_{2, D, m}$ into hyperplanes. The stratification of the critical set in Morin singularities still applies, but there are no results at this point about the global topology of the finer strata.

The idea behind the construction of the homotopy is to change $u$ so as to approach a function $u_{*}$ which is constant equal to a value $x_{m}$ with the property that $f^{\prime}\left(x_{m}\right)=-m^{2}$ except for two small intervals at the endpoints. Notice that the (fundamental) solution of

$$
-v^{\prime \prime}-m^{2} v=0, \quad v(0)=0, v^{\prime}(0)=1
$$

is the function $v(t)=\sin (m t) / m$. In general, define the m-argument $\omega_{m}(t)$, the argument of the vector $\left(v_{1}^{\prime}(t), m v_{1}(t)\right)$. Since $\omega(t)=\omega_{m}(t)$ if $\omega(t)=k \pi$, $k \in \mathbb{Z}$, the sets $C_{2, D, m}$ are $m \pi$-levels for both $\omega(\pi)$ and $\omega_{m}(\pi)$. Better still, the $m$-argument of $v(t)=\sin (m t) / m$ varies linearly from 0 to $m \pi$ for $t \in[0, \pi]$. In figure 3 we show an example of $u \in C_{2, D, m}, m=2$, and in dotted lines the constant value $x_{m}$; below we show the $m$-argument $\omega_{m}$ of $u$ and the constant function $x_{m}$.

The homotopy squeezes the graph of $\omega_{m}$ between parallel walls advancing towards the line $y=m t$, as shown in figure 4. A corresponding $u$ is obtained by changing its original value in the region of the domain over which the graph of $\omega_{m}$ has been squeezed - there, the new value of $u$ is $x_{m}$. The value of $\omega_{m}(\pi)$ for this new $u$ equals $m \pi$, but such $u$ is discontinuous and therefore not acceptable. We make amends: the region where the graph of $\omega_{m}$ trespasses the wall by more than a prescribed tolerance is taken to $x_{m}$ and in the region where the graph of $\omega_{m}$ lies strictly between the walls, $u$ is unchanged. Hence, there is an open region in the domain where $u$ assumes rather arbitrary values in order to preserve its continuity. 

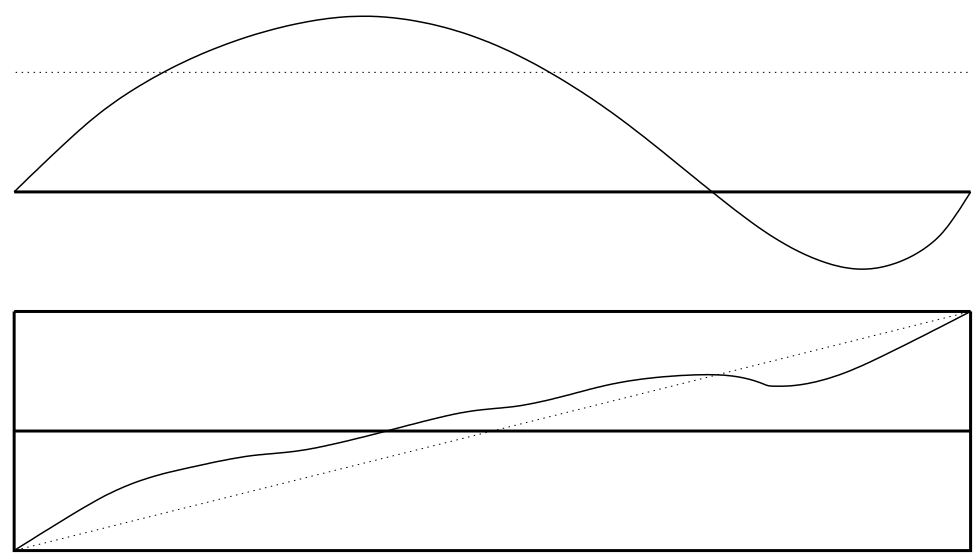

Figure 3: Graphs of $u$ and $x_{m}$ and their $m$-arguments
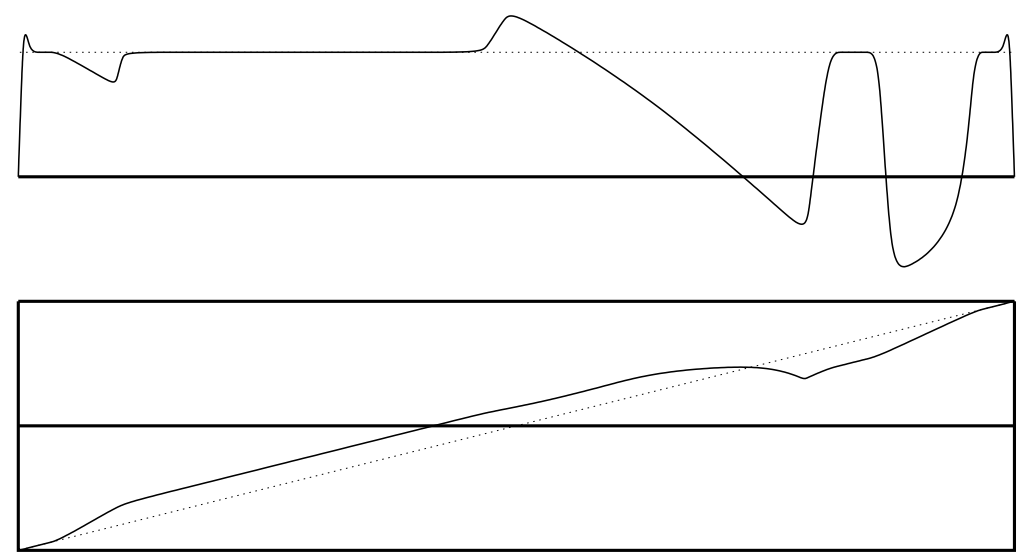

Figure 4: The function $u$ gets squeezed

Similar results were previously obtained for the special case when $f$ is a convex nonlinearity $([15], 3])$. The trivial topology of each connected component $C_{2, D, m}$ then follows from the fact that each such set is a graph over the hyperplane in $H_{D}^{2}$ of functions orthogonal to $\sin t$.

\section{The second order periodic case}

Things get more complicated for the second order operator with periodic boundary conditions. As is well known, the associated linearizations do not necessarily have simple spectrum. Critical sets now are not submanifolds, and one has to be careful about nonregular points. Still, a description of the global geometry of the critical set is possible ([5] $)$. 
For any smooth nonlinearity $f: \mathbb{R} \rightarrow \mathbb{R}$ denote by $F_{2, p}$ the differential operator

$$
\begin{aligned}
F_{2, p}: H_{p}^{2} & \rightarrow L^{2} \\
u & \mapsto-u^{\prime \prime}+f(u)
\end{aligned}
$$

where $H_{p}^{2}=H_{p}^{2}([0,2 \pi] ; \mathbb{R})=H^{2}\left(\mathbb{S}^{1} ; \mathbb{R}\right)$ and $L^{2}=L^{2}([0,2 \pi] ; \mathbb{R})=L^{2}\left(\mathbb{S}^{1} ; \mathbb{R}\right)$. We are interested in the critical set $C_{2, p} \subset H_{p}^{2}$ of $F_{2, p}$. Again, the differential of $F_{2, p}$ is the Fredholm linear operator $D F_{2, p}(u) v=-v^{\prime \prime}+f^{\prime}(u) v$ of index 0 .

Let $\Sigma_{0} \subset \mathbb{R}^{3}$ be the plane $z=0$ and, for $n>0$, let $\Sigma_{n}$ be the cone

$$
x^{2}+y^{2}=\tan ^{2} z, \quad 2 \pi n-\frac{\pi}{2}<z<2 \pi n+\frac{\pi}{2}
$$

and $\Sigma=\bigcup_{n \geq 0} \Sigma_{n}$

Theorem 7 Let $f: \mathbb{R} \rightarrow \mathbb{R}$ be smooth function such that $f^{\prime}$ has isolated critical points and $f^{\prime}$ is surjective. Then the pair $\left(H_{p}^{2}, C_{2, p}\right)$ is diffeomorphic to the pair $\left(\mathbb{R}^{3} \times \mathbb{H}, \Sigma \times \mathbb{H}\right)$.

The set $C_{2, p}^{*} \subset C_{2, p}$ of potentials $u$ for which all solutions $v$ of

$$
-v^{\prime \prime}(t)+f^{\prime}(u(t)) v(t)=0
$$

are periodic is the set of nonregular points. It follows from theorem 7 that $C_{2, p}^{*}$ is a submanifold of codimension 3 , taken by the diffeomorphism mentioned in the theorem to the set vertices of the cones, i.e., to points of the form $((0,0,2 \pi n), *) \in$ $\mathbb{R}^{3} \times \mathbb{H}$.

The proof of theorem [7 requires the topological study of the monodromy map. For $h \in L^{2}$, let $v_{1}, v_{2} \in H^{2}([0,2 \pi] ; \mathbb{R})$ be defined by

$$
v_{i}^{\prime \prime}(t)=h(t) v_{i}(t), \quad v_{1}(0)=1, v_{1}^{\prime}(0)=0, v_{2}(0)=0, v_{2}^{\prime}(0)=1
$$

and define $\tilde{\beta}:[0,2 \pi] \rightarrow G$ by $\tilde{\beta}(0)=I$ and

$$
\tilde{\beta}(t)=\left(\begin{array}{ll}
v_{1}(t) & v_{1}^{\prime}(t) \\
v_{2}(t) & v_{2}^{\prime}(t)
\end{array}\right)
$$

where $G=\widetilde{S L(2, \mathbb{R})}$ is the universal cover of $S L(2, \mathbb{R})$. Finally, define $\xi_{\bullet}: L^{2} \rightarrow G$ by $\xi_{\bullet}(h)=\tilde{\beta}(2 \pi)$. The map $\xi_{\bullet}$ is not surjective: its image is an open set $G_{+} \subset G$ diffeomorphic to $\mathbb{R}^{3}$. The map $\xi_{\bullet}$ is in a sense a projection:

Proposition 6.1 There exists a smooth diffeomorphism $\Psi_{k}: G_{+} \times \mathbb{H} \rightarrow L^{2}$ such that $\xi_{\bullet} \circ \Psi_{k}$ is the projection on the first coordinate.

Further results in infinite dimensional topology are needed to handle the nonregular points. 


\section{$7 \quad$ The third order case}

In the second order periodic case, the set $C_{2, p}^{*}$ plays a very important role. For the harder third order case, it is natural to start by considering its counterpart, the set $C_{3, p}^{*} \subset\left(H^{3}\left(\mathbb{S}^{1}\right)\right)^{2}$ of pairs of potentials $\left(h_{0}, h_{1}\right)$ for which all solutions $v$ of

$$
v^{\prime \prime \prime}(t)-h_{1}(t) v^{\prime}(t)-h_{0}(t) v(t)=0
$$

are periodic, i.e., satisfy

$$
v(0)=v(2 \pi), \quad v^{\prime}(0)=v^{\prime}(2 \pi), \quad v^{\prime \prime}(0)=v^{\prime \prime}(2 \pi) .
$$

For $\left(h_{0}, h_{1}\right) \in C_{3, p}^{*}$, let $v_{0}, v_{1}, v_{2}$ be the (fundamental) solutions of $(\dagger$ ) with initial conditions

$$
\left(\begin{array}{ccc}
v_{0} & v_{1} & v_{2} \\
v_{0}^{\prime} & v_{1}^{\prime} & v_{2}^{\prime} \\
v_{0}^{\prime \prime} & v_{1}^{\prime \prime} & v_{2}^{\prime \prime}
\end{array}\right)(0)=I
$$

and normalize:

$$
\gamma(t)=\frac{1}{\left|\left(v_{0}(t), v_{1}(t), v_{2}(t)\right)\right|}\left(v_{0}(t), v_{1}(t), v_{2}(t)\right) .
$$

A straightforward computation yields

$$
\operatorname{det}\left(\gamma(0), \gamma^{\prime}(0), \gamma^{\prime \prime}(0)\right)=1, \quad \operatorname{det}\left(\gamma(t), \gamma^{\prime}(t), \gamma^{\prime \prime}(t)\right)>0 \text { for all } t .
$$

Conversely, a curve $\gamma:[0,2 \pi] \rightarrow \mathbb{S}^{2}$ is locally convex if $\operatorname{det}\left(\gamma(t), \gamma^{\prime}(t), \gamma^{\prime \prime}(t)\right)>0$ for all $t$. Notice that this implies $\gamma^{\prime}(t) \neq 0$ for all $t$. Let $X_{I}$ be the set of locally convex curves $\gamma$ (with appropriate smoothness hypothesis) satisfying

$$
\gamma(0)=\gamma(2 \pi)=e_{1}, \quad \gamma^{\prime}(0)=\gamma^{\prime}(2 \pi)=e_{2}, \quad \gamma^{(j)}(0)=\gamma^{(j)}(2 \pi)
$$

and let $X_{I}^{1} \subset X_{I}$ be the set of curves $\gamma$ with $\operatorname{det}\left(\gamma(0), \gamma^{\prime}(0), \gamma^{\prime \prime}(0)\right)=1$. Clearly, the inclusion $X_{I}^{1} \subset X_{I}$ is a homotopy equivalence. The map just described from $C_{3, p}^{*}$ to $X_{I}^{1}$ is a diffeomorphism; we proceed to construct its inverse. Given $\gamma \in X_{I}^{1}$, set

$$
r(t)=\left(\operatorname{det}\left(\gamma(t), \gamma^{\prime}(t), \gamma^{\prime \prime}(t)\right)\right)^{-1 / 3}
$$

and $V(t)=r(t) \gamma(t)$. We then have $\operatorname{det}\left(V(t), V^{\prime}(t), V^{\prime \prime}(t)\right)=1$ for all $t$, which implies that the vector $V^{\prime \prime \prime}(t)$ is a linear combination of $V(t)$ and $V^{\prime}(t)$. In other words, there exist unique real valued functions $h_{0}$ and $h_{1}$ with $V^{\prime \prime \prime}(t)=$ $h_{0}(t) V(t)+h_{1}(t) V^{\prime}(t)$, so that each coordinate of $V$ is a periodic (fundamental) solution of $(\dagger)$ and the pair $\left(h_{0}, h_{1}\right)$ belongs to $C_{3, p}^{*}$. Thus, $C_{3, p}^{*}$ is homotopy equivalent to $X_{I}$.

The space $X_{I}$ has been studied, among others, by Little ([9]), B. Shapiro, M. Shapiro and Khesin $\left(\left[19\right.\right.$, [20], [18]). Little showed that $X_{I}$ has three connected components which we shall call $X_{-1, c}, X_{1}$ and $X_{-1}$. One of the authors 
(S., [16]) established several results concerning the homotopy and cohomology of these components. It turns out that $X_{-1, c}$ is contractible and $X_{1}$ and $X_{-1}$ are simply connected but not homotopically equivalent to finite $\mathrm{CW}$-complexes. Also, $\pi_{2}\left(X_{-1}\right)$ contains a copy of $\mathbb{Z}$ and $\pi_{2}\left(X_{1}\right)$ contains a copy of $\mathbb{Z}^{2}$. As for the cohomology, $H^{n}\left(X_{1}, \mathbb{R}\right)$ and $H^{n}\left(X_{-1}, \mathbb{R}\right)$ are nontrivial for all even $n$, $\operatorname{dim} H^{4 n-2}\left(X_{1}, \mathbb{R}\right) \geq 2$ and $\operatorname{dim} H^{4 n}\left(X_{-1}, \mathbb{R}\right) \geq 2$ for all positive $n$.

These results indicate that the topology of the critical set of third order operators is far more complicated than the lower order counterparts.

\section{References}

[1] Ambrosetti, A. and Prodi, G., On the inversion of some differentiable maps between Banach spaces with singularities, Ann. Mat. Pura Appl. 93, 231246, 1972.

[2] Berger, M. and Podolak, E., On the solutions of a nonlinear Dirichlet problem, Ind. Univ. Math. J. 24, 837-846, 1975.

[3] Bueno, H. and Tomei, C., Critical sets of nonlinear Sturm-Liouville operators of Ambrosetti-Prodi type, Nonlinearity 15 (2002) 1073-1077.

[4] Burghelea, D., Saldanha, N. and Tomei, C., Results on infinite-dimensional topology and applications to the structure of the critical set of nonlinear Sturm-Liouville operators, J.Diff.Eq, 188, 569-590, 2003.

[5] Burghelea, D., Saldanha, N. and Tomei, C., The topology of the monodromy map of the second order ODE and the structure of the critical set of nonlinear Sturm-Liouville operators, in preparation.

[6] Cafagna, V. and Donati, F., Un résultat global de multiplicité pour un probléme différentiel non linéaire du premier ordre, C. R. Acad. Sci. Paris Sér. 300, 523-526 (1985).

[7] Church, P. T., Dancer, E. N. and Timourian, J. G., The structure of a nonlinear elliptic operator, Trans. Amer. Math. Soc., 338, no. 1 (1993), $1-42$.

[8] Lins Neto, A., On the number of solutions of the equation $\frac{d x}{d t}=$ $\sum_{j=0}^{n} a_{j}(t) x^{j}, 0 \leq t \leq 1$, for which $x(0)=x(1)$, Inventiones Math., 59 (1980), 67-76.

[9] Little, J. A., Nondegenerate homotopies of curves on the unit 2-sphere, J. Differential Geometry, 4, 339-348, 1970. 
[10] McKean, H.P. and Scovel, J.C., Geometry of some simple nonlinear differential operators, Ann. Sc. Norm. Sup. Pisa Cl. Sci. (4) 13, 299-346 (1986).

[11] Malta, I., Saldanha, N. C. and Tomei, C., The numerical inversion of functions from the plane to the plane, Math. Comp.65, 1531-1552, 1996.

[12] Malta, I., Saldanha, N. C. and Tomei, C., Morin singularities and global geometry in a class of ordinary differential equations, Top. Meth. in Nonlinear Anal., 10 (1), 137-169, 1997.

[13] Malta, I., Saldanha, N. C. and Tomei, C., Regular level sets of averages of Nemytskiĭ operators are contractible, J. Func. Anal., 143, 461-469, 1997.

[14] Morin, B., Formes canoniques de singularités d'une application différentiable, C. R. Acad. Sc. Paris, 260 (1965), 5662-5665 and 6503-6506.

[15] Ruf, B., Singularity theory and bifurcation phenomena in differential equations, Quaderno 19, Univ. Studi Milano, 1996.

[16] Saldanha, N., Homotopy and cohomology of spaces of locally convex curves in the sphere, arXiv:math.GT/0407410.

[17] Saldanha, N. and Tomei, C., Functions from $\mathbb{R}^{2}$ to $\mathbb{R}^{2}$ : a study in nonlinearity, arXiv:math.NA/0209097.

[18] Shapiro, B. and Khesin, B., Homotopy classification of nondegenerate quasiperiodic curves on the 2-sphere, Publ. Inst. Math. (Beograd) 66(80), 127-156, 1999 .

[19] Shapiro, B. and Shapiro, M., On the number of connected components of nondegenerate curves on $\mathbb{S}^{n}$, Bull. of the AMS, 25, 75-79, 1991.

[20] Shapiro, M., Topology of the space of nondegenerate curves, Math. USSR, 57, 106-126, 1993.

[21] Whitney, H., On singularities of mappings of Euclidean spaces I. Mappings of the plane into the plane, Ann. Math., 62 (1955), 374-410.

Nicolau C. Saldanha and Carlos Tomei, Departamento de Matemática, PUC-Rio R. Marquês de S. Vicente 225, Rio de Janeiro, RJ 22453-900, Brazil

nicolau@mat.puc-rio.br; http://www.mat.puc-rio.br/ nicolau/

tomei@mat.puc-rio.br 